\title{
CONSTRUCCIÓN COLECTIVA DE UN CONCEPTO DE CUIDADO HUMANIZADO EN ENFERMERÍA ${ }^{1}$
}

\section{COLLECTIVE BUILDING OF A CONCEPT IN NURSING HUMANIZED CARE}

\author{
Ángela Espinosa Aranzales * \\ Carolina Enríquez Guerrero** \\ Felipe Leiva AranZalez ${ }^{* * *}$ \\ Martha López ArÉVALO ${ }^{* * * *}$ \\ LuZ CastaÑEDa RodRÍGUeZ ${ }^{* * * *}$
}

\begin{abstract}
RESUMEN
Objetivo: Construir colectivamente un concepto de cuidado humanizado de enfermería en dos hospitales universitarios de Bogotá D.C. Método: Investigación cualitativa, dialéctica participativa de enfoque histórico-hermenéutico. Se hicieron ocho grupos focales y una entrevista semiestructurada para un total de 57 profesionales de enfermería. La construcción se hizo a partir de un preconcepto basado en el marco teórico, el cual se sometió a discusión entre los participantes. El análisis de los datos se hizo a través de la metodología de análisis de contenido. Resultados: El concepto final incluyó seis categorías: integralidad del sujeto, dignidad y condición humana, principios éticos, reflexión científica, autocuidado y comunicación asertiva y efectiva. Éste reúne elementos fundamentales para la expresión del acto de cuidar, lo que puede sugerir que el atributo de humanización está implícito en el cuidado de enfermería. Conclusión: Se aprecia una concepción operativa y limitada que no evidencia una reflexión trascendente sobre el significado del hombre y la humanización. Los hallazgos de esta investigación sirvieron como base para el diagnóstico situacional del cuidado humanizado en las instituciones objeto de estudio, así como para la búsqueda de alternativas para el fortalecimiento de la humanización como un sello en los servicios de enfermería.
\end{abstract}

Palabras clave: Cuidado de enfermería, humanización de la atención, enfermería.

\begin{abstract}
Objective: The aim of this study was build a concept of humanized nursing care a collectively way in two university hospitals in Bogotá DC. Method: It is a participatory dialectic qualitative research with an historical hermeneutic approach. Eight focus groups and one semi-structured interview were made for a total of 57

\footnotetext{
${ }^{1}$ Investigación Subsidiada por el Fondo de Investigaciones de la Universidad del Rosario-FIUR Bogotá D.C.

* Investigadora Principal. Enfermera, magíster en epidemiología. Profesora Principal Escuela de Medicina y Ciencias de

** Enfermera, magíster en enfermería y en epidemiología. Instructora de Práctica Escuela de Medicina y Ciencias de la

${ }^{* * *}$ Filósofo. Investigador de Proyecto. Escuela de Medicina y Ciencias de la Salud. Universidad del Rosario. Bogotá D.C.

${ }^{* * * *}$ Enfermera, especialista en gerencia hospitalaria. Gestora de Enfermería Méderi. Bogotá D.C. Colombia. Email:

${ }^{* * * * *}$ Enfermera, especialista en epidemiología. Coordinadora Educación en Enfermería Méderi. Bogotá D.C. Colombia.
} la Salud. Universidad del Rosario. Bogotá D.C. Colombia. Email: angela.espinosa@urosario.edu.co Salud. Universidad del Rosario. Bogotá D.C. Colombia. Email: carolinal.enriquez@urosario.edu.co Colombia. Email: felipeleivaaranzalez@gmail.com mrosbita@hotmail.com Email: luzadricr07@yahoo.com
\end{abstract}


nurses. The construction was based on pre-concept took from theoretical framework, which was submitted for discussion among participants. The data analysis was done through content analysis methodology. Results: The final concept included six categories: integrity of the subject, dignity and human condition, ethical principles, scientific reflection, and assertive effective communication. It brings key elements for expression of the act of caring which may suggest that the humanized attribute is implicit in nursing care. Conclusion: This operational and limited conception no evidences a transcendent reflection about meaning of human being and humanization. The findings of this research were the basis for one diagnosis of humanized nursing care in institutions under consideration and for the search of alternatives to strengthen the humanization as a label in nursing services.

Key words: Nursing care, humanization of assistance, nursing.

Fecha recepción: 10/04/14 Fecha aceptación: 10/04/15

\section{INTRODUCCIÓN}

La relación del hombre consigo mismo y con los otros ha sido eludida y soslayada en el estadio de civilización actual debido, entre otras razones, a una crisis en cuanto a su propia autodeterminación (1), su autoconocimiento (2) y su propia valoración a nivel de sus relaciones sociales y culturales; este permanecer en el mundo "fuera de sî" conduce al hombre a transformarse en un medio para llegar a fines ajenos a él y no como lo postula Kant, en una de las fórmulas de su imperativo categórico, tratar al hombre como si fuera un fin en sí mismo (3).

Para corrientes de pensamiento marxistas no ortodoxas $(1,4,5)$, la forma de producción de mercancías dentro de la economía de mercado enajena al ser humano, debido a que dentro de dicha economía la principal preocupación se centra en las "cosas" del mundo, las que se crean con la "fuerza de trabajo" del hombre pero que no le pertenecen a éste y que adquieren un valor de cambio en el entramado mercantil. De esta forma su humanidad puede verse reducida a la concepción de "cosa" y al valor que como cosa tiene en el conjunto de actividades económicas; así el hombre escindido por la determinación socioeconómica se convierte en una especie de herramienta, instrumento o medio y en el campo de las relaciones humanas este "proceso de cosificación del hombre" (4) puede llamarse deshumanización.

La deshumanización ha afectado a las profesiones (6) de la salud, la disciplina profesional de enfermería no escapa a esta realidad; como lo afirma Romero, la relación enfermera-sujeto de cuidado se ha viciado, se ha "invisibilizado", el ejercicio de enfermería hoy está centrado principalmente en funciones administrativas que obedecen a exigencias del mercado del sistema de la salud, pasando a un segundo o tercer plano la relación interpersonal sujeto de cuidado-enfermera (7). Además, Watson afirma que aunque el cuidado es la esencia de la práctica de enfermería, éste ha estado perdiendo protagonismo en los sistemas de salud, principalmente debido a avances en la tecnología y a barreras institucionales (8).

La deshumanización en la profesión de enfermería adquiere diferentes facetas, las cuales en el campo del área asistencial se pueden manifestar fundamentalmente en cuatro aspectos: El primero tiene que ver con el actual modelo de prestación de servicios, determinado por el gran sistema de relaciones económicas, que al priorizar la relación costo-beneficio hace del ejercicio profesional una carga desgastante, limita la actividad creativa $(7,9-12)$ y favorece la deshumanización de las relaciones laborales. 
El segundo se refiere a que los enfermeros en su relación con otras disciplinas de la salud y entre sí, en muchos casos alteran sus relaciones profesionales $(7,10-12)$ ya sea por la búsqueda de reconocimiento, por una competitividad desenfocada o por una forma adaptativa perversa de sobrellevar las exigencias de cumplimiento del sistema económico de la salud. El tercer aspecto tiene que ver con la limitada relación entre enfermeros y pacientes $(7,11,12)$, en la cual el enfermero diluye o delega su quehacer fundamental, responder a las necesidades de cuidado del sujeto, y la humanidad se desvirtúa como un mero hecho que es apenas considerado en el complejo sistema actual de prestación de servicios.

El cuarto aspecto, además de una faceta es una consecuencia, tiene que ver con la pérdida de autonomía $(9,12)$ en el ejercicio de la enfermería; el profesional está supeditado a las demandas del sistema económico y al estar alejado del sujeto de cuidado no puede evidenciar el impacto intrínseco de su quehacer centrando su ejercicio en actividades dependientes de otras disciplinas.

Como consecuencia de lo anterior se puede afirmar que el ejercicio profesional de enfermería entra en un ciclo inicuo de deshumanización: el alejamiento del sujeto de cuidado, la desvirtuación de su ejercicio profesional y la pérdida de autonomía lo hacen vulnerable; las exigencias del sistema económico que no pueden apreciar un lucro tangible en el actuar de enfermería vulneran la profesión y los profesionales vulnerados en un afán equívoco de "cumplirle" al sistema y de "adaptarse" a él terminan vulnerando al sujeto de cuidado, ya sea con un trato no digno o con un contacto limitado.

Autoras desde sus trabajos sobre el metaparadigma de enfermería $(13,14)$ han propuesto que el cuidado es uno de los elementos nucleares que construye tanto el pensamiento como la práctica enfermera, así mismo Watson expone en uno de los 11 supuestos de los valores del cuidado humano que "el cuidado es el elemento central unificador de la práctica enfermera, la esencia de la enfermería" (8). Así mismo la Ley Deontológica de Enfermería de Colombia expone que "El acto de cuidado de enfermería es el ser y esencia de la profesión" (15), manifestado como una relación única e interpersonal.

Para responder a la condición del ser del paciente, condición fundamental para el cuidado, es preciso que el enfermero entre a la realidad subjetiva de quien cuida. Newman refiere que el cuidado es uno de los elementos definitorios de enfermería y fundamental para la identidad de la disciplina, el cuidar potencia una relación transformadora y de calidad entre enfermera y paciente (14). Romero afirma que la relación de la enfermera con los sujetos de cuidado es de forma dialógica (16), donde el contacto directo entre las dos partes es fundamental para el cuidado. Estas afirmaciones están en consonancia con un consenso de unidad filosófica sobre las bases ontológicas de la enfermería que afirman que el ser humano se caracteriza por la integridad, la complejidad y la conciencia; la esencia de enfermería implica un proceso comprometido de humano a humano y la esencia de la práctica de enfermería es la relación enfermera-paciente (17).

De esta forma, el cuidado humanizado en enfermería debería tener en cuenta la humanidad de los sujetos que se relacionan y contemplarse dentro de la estructura significativa que históricamente ha adquirido la humanidad, como especie capaz de creación y entendimiento simbólico, integrada y determinada por las dimensiones psicológica, espiritual, estética, social, política y cultural. El cuidado humanizado demanda el conocer a quien se cuida desde lo humano, el cuidado propuesto tiene significado para la persona, si la enfermera está presente en la relación (18).

De tal manera, el cuidado humanizado ha de presentarse de manera dialógica, como una relación de ida y vuelta entre los sujetos que se relacionan en el cuidado enfermero 
(sujeto de cuidado y cuidador); por lo tanto, su fundamento descansa en el entramado de significados que ha adquirido la humanidad y que convergen en las interrelaciones que suponen las acciones de enfermería y por las cuales adquiere sentido ella misma. Así, el cuidado humanizado en enfermería es una forma de comprender el universo enfermero y su relación epistemológica con el cuidado.

El cuidado humanizado en enfermería debe entenderse dentro de la dimensión simbólica que define al hombre, dado que es por y en medio de éste que los sujetos logran construir un mundo pleno de significado y comprensión.

A base de lo mencionado anteriormente se está ante una contradicción paradójica: por un lado la enfermería expresada en el acto de cuidado presupone la relación directa entre seres humanos, pero por el otro se tiene una desvirtuación multicausal del ejercicio profesional: organización de la prestación de los servicios de salud centrado en la productividad económica, primacía de la tecnología sobre lo humano, falta de apropiación del rol del enfermero, entre otros.

Este trabajo se inscribe dentro de un proceso de investigación de largo alcance que tuvo por objeto encontrar alternativas que promocionaran y fortalecieran el cuidado humanizado en los servicios de enfermería de dos instituciones hospitalarias universitarias en Bogotá D.C. Metodológicamente hay un primer momento de conceptualización, seguido de un diagnóstico y posteriormente la búsqueda de las alternativas.

Inicialmente se buscó un concepto de cuidado humanizado que se adaptara a la realidad del ejercicio de enfermería en la ciudad de Bogotá. Sin embargo no hubo una noción única y significativa para el contexto de estudio. Las publicaciones encontradas presentan una mirada operativa de la humanización centrada en la medición de conductas necesarias para asegurar un servicio con calidad como un principio de humanización (19); lo muestran desde una mirada ética y moral
(20); como un cambio en la cultura organizacional hacia el reconocimiento subjetivo e histórico tanto de los clientes como de los trabajadores de la salud (21).

Desde esta perspectiva surgió la necesidad de construir colectivamente un concepto de cuidado, en el que la humanización fuera el derrotero que conjugara las dos posiciones de la paradoja mencionada anteriormente y que expresara lo manifestado por la Ley Deontológica y por las teóricas del cuidado en sus postulados.

\section{MÉTODO}

Es una investigación cualitativa "dialéctica participativa" de enfoque histórico-hermenéutico. El componente dialéctico participativo se evidenció en la reflexión sobre la práctica enfermera a la luz de la teoría y una validación y enriquecimiento de la teoría a la luz de la práctica. Se les dio voz activa a los sujetos de conocimiento, dado que ellos son los que ejercen la práctica, la cual fue el insumo base para este estudio.

El enfoque de la investigación se basó en los trabajos realizados por Dilthey (22), Ricoeur (23) y Gadamer (24). Se tuvo en cuenta las estructuras de sentido intersubjetivamente compartidas, ya que existe una preconcepción de lo que es el cuidado humanizado que está dotado de sentido (22) y por lo mismo, fue en la interacción con los enfermeros donde el concepto obtuvo las condiciones de posibilidad que le permitieron una determinación significativa tanto para los sujetos de investigación como para la investigación misma.

La población a estudio fueron profesionales de enfermería de dos instituciones hospitalarias universitarias de Bogotá D.C. Los criterios de inclusión fueron: los participantes debían ser profesionales y tener como mínimo tres meses de experiencia laboral en cada una de las instituciones. En el trabajo 
de campo se contó con la participación de 57 enfermeros. 50 mujeres y 7 hombres en edades entre los 20 y 55 años, formados en universidades públicas y privadas de Colombia. Se formaron ocho grupos focales conformados por mínimo cinco y máximo diez participantes y una entrevista semiestructurada.

Los datos arrojados por esta investigación, como en la mayoría de las investigaciones de corte cualitativo, son texto y, como tal, son susceptibles de interpretación. Ésta pone de manifiesto la multiplicidad de matices y la rica posibilidad de que sean interpretados, por lo tanto, comprender y explicar la riqueza del hecho narrado en texto implica posicionarse históricamente frente al fenómeno para poder comparar y observar los posibles quiebres, las tensiones y posibilidades que ofrece en sí el dato (23).

Dada la inconstancia de lo teórico en el plano del ejercicio práctico del cuidado, los investigadores abordaron el problema de la construcción del concepto de cuidado humanizado planteando, en principio, un concepto predeterminado basado en el marco teórico del proyecto, buscando una proyección crítico-reflexiva por parte de los participantes sobre el mismo.

El análisis de los datos se hizo a través del análisis narrativo de contenido (25); para la interpretación de los resultados se hizo una división de las categorías entre los investigadores, luego cada uno leía lo trabajado por el otro para identificar coincidencias y diferencias con el fin de unificar la interpretación.

Dado el número de grupos focales (ocho) y la entrevista se pudo obtener una cantidad de datos suficiente sobre las diferentes categorías de análisis, así mismo se encontró la repetición de ideas tanto en el trabajo de campo como en su reproducción en las transcripciones, lo cual llevó a establecer el grado de saturación del dato en esta primera etapa de investigación, permitiendo recrear un nuevo concepto de cuidado humanizado, cuidando de no tener un enfoque muy restringido y de hacer las preguntas pertinentes sobre el tema (26).

Puede sugerirse que a partir de la metodología dialéctica, donde se entregaba teoría sobre humanización a los participantes, los datos obtenidos, aunque pudieron ser permeados por la información previamente entregada, en su mayoría provinieron de la práctica misma en sus actividades hospitalarias cotidianas; así el movimiento de los datos fue de ida y vuelta, en tanto la teoría fue útil como un apoyo para que los participantes hicieran discursiva su práctica clínica.

Según la Resolución 8430 de 1993 del Ministerio de Salud Colombiano (27) que fija las normas para la investigación con sujetos humanos, se considera que esta investigación fue de bajo riesgo. Se contó con la aprobación del Comité de Ética en Investigaciones de la Universidad del Rosario y de cada uno de los comités de investigaciones de las instituciones hospitalarias.

\section{RESULTADOS}

La definición del concepto Cuidado humanizado, preelaborado por los investigadores a base de la revisión de la literatura $(4,15,21$, 28-31), se presenta a continuación: "Cuidado humanizado en enfermería es el acto de cuidar al individuo, la familia y la colectividad a partir del reconocimiento del otro como igual a mí, en cuanto a su dignidad de ser humano, reflexionando acerca de sus necesidades de salud, de cuidado y de sus respuestas a esas necesidades, asistiéndolo a partir del respeto por los imperativos éticos en aquéllas en las que se encuentre deficitario, promoviendo dentro de las posibilidades la recuperación pronta de su autocuidado".

En el análisis hecho por los participantes al concepto preelaborado por los investigadores se pudieron identificar tres tendencias y dos puntos emergentes; en la primera tendencia algunos participantes estaban de acuerdo con lo planteado, llamó la atención 
una afirmación que consideró que el concepto reunía atributos necesarios para ser validado:

La opinión con relación a la definición de cuidado humanizado me parece que es acertada, es clara, es integral.

En la segunda tendencia algunos participantes complementaron el concepto. En primera instancia respecto a la importancia de ver al sujeto de cuidado de manera holística, ya que se reconoce que la enfermedad está condicionada no sólo por las manifestaciones corporales:

Entendiendo que el individuo no solo está enfermo de salud, sino que también tiene unos componentes religiosos, sociales, económicos que lo afectan...

En segunda instancia complementaron el concepto mostrando la relevancia de la sensibilidad del enfermero frente al sujeto de cuidado:

La sensibilidad que tiene de pronto, hacia el cuidado con el paciente, o el amor con el que realiza las cosas.

Por último mostraron la importancia del componente comunicacional que debe tener el concepto. Si bien no se hizo una alusión directa en las intervenciones expresadas, se puede asumir como tal a base de las opiniones dadas por los participantes:

Yo le explico todo al paciente para que se sienta seguro, y eso es cuidado humanizado.

Son cosas que uno puede decir en un momento dado quizás muy rápidas pero oportunas, para que la persona pierda ya su tensión, su intranquilidad y uno como que pueda dar esa atención más cálida.

La tercera tendencia cuestionó algunos apartes del concepto. Primero en lo que res- pecta al reconocimiento del otro como igual a mí:

Y yo pensaría que mi atención no puede estar condicionada a alguna cosa, a alguna relación de afecto, no, cuando yo estudié y tomé esta decisión del cuidado del paciente fue libre y no tengo por qué condicionarlo.

En segunda instancia se cuestionó lo relativo a los imperativos éticos:

Me parece que imperativos éticos quedaría como muy macro, muy general, que tendríamos que desglosar o ser más minuciosos con el término.

El concepto me parece bastante completo, si comparto lo que dice el jefe con relación a los imperativos, no sé si es necesario cambiar, buscar un significado con relación a esta parte, porque como que lo trata de desorientar a uno en alguna oportunidad...

En cuanto a los puntos emergentes el primero equipara el concepto de cuidado humanizado presentado con el concepto de enfermería:

Digo yo que ahí nos están describiendo, es lo que nosotros le vamos a aportar a nuestro paciente, a nuestro familiar, es nuestro deber ser.

Y el segundo punto emergente manifestó una diferencia entre el cuidado personalizado y el cuidado humanizado:

Una cosa es el cuidado humanizado y otra el cuidado personalizado, yo puedo tener $40 \mathrm{pa}$ cientes pero puedo ser humanizado con todos.

A base de lo propuesto por los participantes a partir del preconcepto presentado se mantuvo lo aceptado por ellos, referente a los apartes: acto de cuidado, reflexión acerca de sus necesidades, autocuidado. Se complementó en cuanto integralidad y comunicación y se modificó lo referente a re- 
conocimiento del otro como igual a mí y los aspectos éticos. De esta forma el concepto construido participativamente entre los sujetos de investigación y los investigadores fue: "Es la expresión del acto de cuidado en la que se concibe integralmente al individuo, la familia y la colectividad; en la que se reconoce la dignidad y condición humana del otro; en la que se respetan los principios éticos; en la que se reflexiona acerca de sus necesidades de cuidado en salud y de sus respuestas a las mismas para asistirlo en aquellas demandas en las que se encuentre deficitario, promoviendo dentro de las posibilidades la recuperación pronta de su autocuidado a través de una comunicación asertiva y efectiva".

\section{DISCUSIÓN Y CONCLUSIÓN}

El concepto construido contiene la idea de un sujeto de cuidado integral, que además de incluir al paciente incluye a su familia y a la colectividad, notándose la importancia de concebir en la relación de cuidado humanizado la figura del "otro", el receptor del cuidado junto con las personas que lo rodean. Los resultados muestran la preocupación de enfermería por concebir al sujeto de cuidado como un ser social, y no solamente como un sujeto afectado en su necesidad de salud; así se pone de manifiesto que el sujeto de atención y su contexto son elementos claves en el ser y esencia de enfermería, como lo demuestran los trabajos que sobre los conceptos metaparadigmáticos ha realizado Fawcett y Newman et al. $(13,14)$.

Para Ricoeur, quien retoma, analiza y critica los trabajos de Dilthey y Gadamer sobre la hermenéutica y la gran importancia que tiene el contexto para llevarla a buen término (23), "la actualización del texto" como dato debe tener como componente fundamental el lugar histórico de su enunciación. Así, puede notarse que los resultados del estudio están atados intrínsecamente con el marco de referencia social que acontece en los diferentes niveles donde el cuidado se expresa, estos niveles, como el institucional o el profesional, permiten observar que el concepto construido trata de reflejar las diferentes problemáticas que socavan las relaciones del cuidado en enfermería.

La importancia de la "sensibilidad" para el cuidado humanizado que destacan los participantes se puede relacionar con Watson, quien en los 10 factores de cuidado resalta la importancia del cultivo de la sensibilidad hacia sí mismo y hacia los demás como un componente estructural del fundamento filosófico para la ciencia del cuidado $(8,32)$.

La comunicación asertiva y efectiva no fue tomada en cuenta en la construcción del preconcepto, sin embargo surge como un componente establecido por los propios participantes, lo cual podría indicar que es un elemento que emerge de la práctica misma de la enfermería, dejando entrever su importancia para la expresión del cuidado humanizado. En consonancia con la literatura que ha resaltado la relevancia de la comunicación en la satisfacción y seguridad del paciente, la adherencia de éste al tratamiento y la promoción del autocuidado $(32,33)$.

En los resultados emerge la dificultad sobre la idea de "reconocimiento del otro" como un igual en cuanto a la dignidad, argumentándose que tal reconocimiento condicionaría el ejercicio profesional ya que el cuidado debería expresarse independientemente de que se logre o no tal reconocimiento. Este desencuentro llama la atención porque, por un lado contraviene la afirmación del cuidado como una relación interpersonal en la que es preciso reconocer en el otro una parte constitutiva del desarrollo de la identidad y, en este caso, de la subjetividad de cuidador con respecto a la subjetividad del ser cuidado tal como lo afirma Honneth al hablar de la necesidad de reconocer en el otro una parte importante para el desarrollo y crecimiento de sí mismo (4); pero por otro lado resalta una clase de independencia en el cuidado, en 
la que, indistintamente de la relación establecida con el que se cuida, éste se debe dar.

Los principios éticos de enfermería direccionan las actividades de la profesión desde el punto de vista del cuidado humanizado, no obstante son principios que rigen a la enfermería sin importar el modelo conceptual que se aplique o desde la perspectiva que se aborde el ejercicio, la legislación de enfermería los incluye como determinantes del cuidado (15, 28). Los participantes los reconocen, sin embargo emerge el desencuentro ante la palabra "imperativos" y la aceptan cuando ésta se cambia por "principios". Aquí se puede apreciar el predominio de lo que "es" (principio) sobre lo que "tiene que ser" (imperativo). Un predominio que podría suponer una afirmación de la libertad en el ejercicio profesional, tal como lo afirma la Ley respecto a que enfermería es una profesión liberal (28).

La promoción del autocuidado es un elemento fundamental en el objeto de enfermería como lo propone Orem en su teoría del "déficit de autocuidado" (8) . Así, hubo consenso en su inclusión en el concepto, lo cual reafirma su importancia desde la práctica. Este componente está relacionado directamente con el principio de autonomía tanto del sujeto de cuidado como del profesional de enfermería. Del sujeto, porque tiene que ver con el reconocimiento de un ser humano que, al estar hospitalizado, se encuentra en un estado de vulnerabilidad, sin embargo por respeto a la autonomía, debe hacerse responsable de su cuidado en la medida de sus posibilidades; tal como lo afirma Swanson, la enfermera debe estar presente para suplir lo que la otra persona no puede hacer por sí misma pero sin afectar su dignidad (18). Del profesional, porque la promoción del autocuidado es una acción independiente de enfermería, para el cual se han desarrollado constructos sólidos y como en toda acción independiente hay una expresión plena y autónoma de los acervos de conocimiento técnico y disciplinar (34).
La reflexión emergente que afirmó que el concepto de cuidado humanizado construido era igual al concepto de enfermería, da pie para suponer en primera instancia, que el atributo de humanizado no es optativo en enfermería; si se falla en éste, se está fallando en el ejercicio de la misma; en segunda instancia que se puede repensar el cuidado humanizado como eje articulador y fundante de enfermería. Esto se encuentra en coherencia con lo afirmado por Swanson respecto a que el cuidado humanizado requiere tener una "amplia experiencia, habilidad y capacidad" en la concepción holística del sujeto en su vida diaria, en su contexto (18), tal como lo demanda el cuidado de enfermería.

El punto emergente que manifestó que cuidado humanizado no es lo mismo que cuidado personalizado permite afirmar que el cuidado directo es un elemento fundamental del cuidado humanizado, aunque no se trata de que la enfermera deba estar todo el tiempo con el paciente sino que a través de la gestión del cuidado dirija y asegure un cuidado directo. Entendiendo gestión del cuidado como "un proceso heurístico, dirigido a movilizar los recursos humanos y los del entorno con la intención de mantener y favorecer el cuidado de la persona que, en interacción con su entorno, vive experiencias de salud" (35) o como un juicio profesional para planear, organizar, motivar y controlar la provisión de cuidados (36).

En consonancia con lo expuesto en la literatura respecto al foco de enfermería (13, 14), el acto de cuidar fue el primer atributo propuesto para la construcción del concepto de cuidado humanizado. Posterior a la discusión, se transformó en la "expresión del acto de cuidado". En esta última acepción se aprecia una dinamización de este aparte, en el que se enfatiza acerca de la importancia de que el cuidado se convierta en acción concreta y no sólo en un proceso abstracto. "El cuidar potencia una relación transformadora y de calidad entre enfermera y paciente" (14). 
Desde el punto de vista metodológico se puede afirmar que los sujetos de investigación tomaron una posición frente a las categorías formuladas en el concepto de cuidado humanizado, es decir que desde su propio lugar de enunciación y de comprensión del mundo (23) sometieron a reflexión las nociones enunciadas. Para algunos su perspectiva, expuesta al contexto, expresa "síntomas" de malestar debido a las dificultades sociales en las que se debe otorgar el cuidado; para otros, el cuidado es un ideal representado en estas categorías y que, por tanto, debería expresarse de esta forma: dificultades sociales versus ideal.

El cuidado se da como el hecho crucial en enfermería, sin embargo en el análisis de los resultados se puede interpretar que los participantes lo asumen de manera genérica y no se manifiesta su posicionamiento desde alguna de las diferentes posturas teóricas existentes como la de Watson y Swanson. En este aspecto epistemológico del cuidado se podría afirmar que los participantes están orientando la práctica de enfermería por un modelo mental construido desde la experiencia personal que expresa la forma como cada enfermera ve a la enfermería. Cuando este modelo prevalece se pone en duda la importancia y la pertinencia del conocimiento disciplinar y por ende la importancia del tipo de práctica que se realiza y se expone a ser vulnerada la autonomía profesional y el bienestar del paciente (37).

$\mathrm{Al}$ ser la enfermería una disciplina profesional (35) en donde el contacto humano es la raíz que sostiene su andamiaje teórico-práctico, como lo demuestran las investigadoras de sus conceptos metaparadigmáticos (13, 14), parece que al construir un concepto de cuidado basado en la humanización del mismo no se tuvo en cuenta, o no fue explicitada, una noción de humanidad reflexionada a partir del contexto y bajo la tutela de una idea de hombre que signifique el desarrollo y recorrido histórico de su estar en el mundo.

Contemplar al "hombre" o a la "persona" bajo alguna de las diferentes miradas que la multiplicidad de modelos teóricos de enfermería propone (35) o desde uno de los enfoques propuestos en la introducción de este artículo $(1,2)$, fue soslayado dada la dificultad de pensar la humanidad en otros términos diferentes a la intuición generada por la práctica diaria en un entorno limitado por los referentes de producción a seguir, como lo afirma Gómez Serrano: "Tenemos que cuidar en circunstancias plenamente desfavorables y en las que el tejido social se ha debilitado" (38).

A pesar de que la humanización, como expresión de características humanas en las relaciones interpersonales, puede ser sometida a diferentes cuestionamientos provocando el disenso en su unificación conceptual, ésta parece ser sobreentendida en el discurso enfermero. La deshumanización de las relaciones sociales y de las relaciones de cuidado enfermero no tuvieron representaciones en el discurso, el pensamiento crítico sobre las circunstancias sociales pareció diluirse en el entramado social y entre los discursos positivistas (1) que recaen desde el sistema de salud y desde la institucionalidad.

La noción propuesta de humanización en el cuidado, lejos de ser tan sólo una serie de actividades operativas y conductuales, está vinculada a una transformación profunda de las formas de ver, oír y comprender el mundo humano (30), donde el cuidado no sólo sea la sumatoria de muchas acciones dirigidas sino un intercambio dialógico entre humanos (7), destacando las características que trascienden la simple biología para llevar a un mayor entendimiento de las esferas del hombre.

Agradecimientos: Los autores agradecen al Fondo de Investigaciones de la Universidad del Rosario (FIUR) por su apoyo y financiación para la realización del proyecto y a las instituciones y profesionales de enfermería que con su participación hicieron posible la realización de este estudio. 


\section{REFERENCIAS}

1. Marcuse H. El hombre unidimensional Ensayo sobre la ideología de la sociedad industrial avanzada. Bogotá: Planeta-Agostini; 1986.

2. Cassirer E. Antropología filosófica: Introducción a una filosofía de la cultura. México: Fondo de cultura económica; 1976.

3. Kant I. Fundamentación de la metafísica de las costumbres. Madrid: Alianza Editorial; 2002. 222 p.

4. Honneth A. Reificación: Un estudio en la teoría del reconocimiento. Buenos Aires: Katz Ediciones; 2007.

5. Horkheimer M. Teoría Crítica. Buenos Aires: Amorrortu Editores; 2003.

6. Coriat B. El taller y el robot: ensayos sobre el fordismo y la producción en masa en la era de la electrónica. Buenos Aires: Siglo Veintiuno; 2004.

7. Romero M. Los cuidados de enfermería en la adversidad. Presencia [Internet]. 2005 ene-jun [citado feb 2014]; 1(1). Disponible en: http://www.index-f.com/ presencia/n1/r11articulo.php

8. Marriner A, Raile M. Modelos y teorías en enfermería. 6ta ed. Barcelona: Elsevier; 2007. 828 p.

9. Malvárez S, Castrillón M. Panorama de la fuerza de trabajo en América Latina. Serie Desarrollo de Recursos Humanos N³9. Washington D.C.: Organización Panamericana de la Salud; 2005. 69 p.

10. Zapata M. ¿Enfermería una profesión en crisis? El caso en la ciudad de MedellínColombia [Tesis Maestría]. [Medellín]: Universidad de Antioquía; 2008. 99 p.

11. Romero M, Mesa L, Galindo S. Calidad de vida de las(os) profesionales de enfermería y sus consecuencias en el cuidado. av.enferm. 2008; 26(2): 59-70.

12. Castrillón M, Orrego S, Pérez L, Ceballos M, Arenas G. La enfermería en Colombia y la reforma en el sector salud. Invest Educ Enferm. 1999; 17(1): 13-33.
13. Fawcett J. Analysis and evaluation of conceptual models. Philadelphia: F.A Davis Company; 1995.

14. Newman M, Sime A, Corcoran-Perry S. The focus of the discipline of nursing. ANS Adv Nurs Sci. 1991; 14(1): 1-6.

15. Congreso de la República de Colombia. Ley 911 de 2004. Bogotá Colombia: Congreso de la República; 2004.

16. Romero M. El cuidado de enfermería en la adversidad: compromisos de resistencia desde la docencia. av.enferm. 2007; 25(2): 112-23.

17. Newman M, Smith M, Dexheimer M, Jones D. The focus of the discipline revisited. ANS Adv Nurs Sci. 2008; 31(1): E1627.

18. Posada M, Mora B. Interpretación de la teoría de Kristen Swanson para un cuidado humanizado. Metas Enferm [Internet]. 2014 [citado jun 2015]; 17(3). Disponible en: http://www.enfermeria21. com/revistas/metas/articulo/80573/

19. Rivera Álvarez LN, Triana Á. Percepción de comportamientos de cuidado humanizado de enfermería en la Clínica del Contry. av.enferm. 2007; 25: 56-68.

20. Poblete Troncoso M, Valenzuela Suazo S. Cuidado Humanizado: Un desafío para las enfermeras en los servicios hospitalarios. Acta paul. enferm. 2007; 20: 499503.

21. Backes DS, Koerich MS, Erdmann AL. Humanizando el cuidado a través de la valoración del ser humano: resignificación de los valores y principios por los profesionales de salud. Rev Lat Am Enfermagem. 2007; 15(1): 34-41.

22. Dilthey W. Dos escritos sobre hermenéutica. El surgimiento de la hermenéutica y los esbozos para una crítica de la razón histórica. Madrid: Istmo; 2000. 247 p.

23. Ricoeur P. Del texto a la acción: ensayos de hermenéutica II. Bueno Aires: Fondo de Cultura Económica de Argentina; 2001.380 p.

24. Gadamer HG. Verdad y método I. Funda- 
mentos de una hermenéutica filosófica. Salamanca: Sígueme; 1977.

25. Vázquez Navarrete MLFdS, R. Mogollón P, A. Fernández de Sanmamed Santos, M. Delgado G., M. Vargas, I. Introducción a las técnicas cualitativas de investigación aplicadas en salud. Barcelona: Servei de publicacions Universitat Autònoma de Barcelona; 2006.

26. Martínez Salgado C. El muestreo en investigación cualitativa: principios básicos y algunas controversias. Cien Saude Colet. 2012; 17(3): 613-9.

27. Ministerio de Salud. Resolución número 8430 de 1993 Por la cual se establecen las normas científicas, técnicas y administrativas para la investigación en salud. Bogotá D.C.: Ministerio de Salud; 1993.19 p.

28. Congreso de la República de Colombia. Ley 266 de 1996. Bogotá Colombia: Secretaría Senado; 1996.

29. J Ferrater Mora Diccionario de Filosofía. Josep-María Terricabras ed. Barcelona: Editorial Ariel, S.A.; 2002. Diccionario de Filosofía; p. 3830.

30. Marcuse H. Un ensayo sobre la liberación. México: Editorial Joaquín Mortiz, S.A.; 1969.94 p.

31. Watson J. Nursing: Human Science and Human Care. A theory of nursing. USA: World Headquarters Jones and Barlett; 1999.
32. Slatore C, Hansen L, Ganzini L, Press $\mathrm{N}$, Osborne $\mathrm{M}$, Chesnutt $\mathrm{M}$, et al. Communication by nurses in the intensive care unit: qualitative analysis of domains of patient-centered care. Am J Crit Care 2012; 21(6): 410-8.

33. Vidal B, Adamuz T, Feliu B. Relación terapéutica: el pilar de la profesión enfermera. Enfermería Global [Internet]. 2009 [citado feb 2014];17. Disponible en: http:// scielo.isciii.es/scielo.php?script=sci_arttext\&pid=S1695-61412009000300021\&l$\mathrm{ng}=\mathrm{es}$

34. Durán VM. Enfermería: Desarrollo teórico e investigativo. Bogotá: Unibiblos; 2001.

35. Kérouac S, Pepin J, Ducharme F, Duquette A, Major F. El pensamiento enfermero. Barcelona: MASSON; 2001-2002. 167 p.

36. Milos P, Larrain A, Simonetti M. Nursing services classification: Proposal to ensure quality care in time of nursing shortages. Cienc enferm. 2009; XV(1): 17-24.

37. Sánchez B. La enfermería como disciplina. En: Díaz L, editor. La investigación y el cuidado en América Latina. Bogotá: Universidad Nacional de Colombia; 2005. p. 15-28.

38. La investigación y el cuidado en América Latina. Bogotá: Universidad Nacional de Colombia Facultad de Enfermería Grupo de Cuidado Unibiblos; 2005. 499 p. 